\section{Transformaciones en los hábitos de comunicación y sociabilidad a través del incremento del uso de redes sociodigitales en tiempos de pandemia}

\author{
Transformations in communication and sociability habits through the \\ increased use of socio-digital networks in times of pandemic
}

\author{
Rubén Cervantes Hernández \\ Universidad Autónoma de Chihuahua | Rúa de las Humanidades S/N, Campus Universitario 1, \\ Chihuahua | México | https://orcid.org/0000-0002-9390-9461| rubencervantesh1@gmail.com
}

\author{
Dra. Paola Margarita Chaparro-Medina \\ Universidad Autónoma de Chihuahua | Rúa de las Humanidades S/N, Campus Universitario 1, \\ Chihuahua | México | https://orcid.org/0000-0002-7270-9903| pchaparro@uach.mx
}

Fechas | Recepción: 10/01/2021 | Aceptación: 27/02/2021

\section{Resumen}

Durante la pandemia que surgió a raíz de la enfermedad COVID-19 se implementaron medidas de seguridad que involucran el confinamiento y distanciamiento de la población. Esto tuvo como consecuencia la modificación de los estilos de vida de los individuos a través de las Tecnologías de la Información y Comunicación (TIC). Para efectos de esta investigación, nos centramos en las redes sociodigitales (RSD), siendo estas de importancia significativa para el desarrollo individual y organizacional. Por ello, se buscó contrastar el uso de diversas aplicaciones, antes y durante la pandemia, por medio de la implementación no probabilística de un cuestionario no longitudinal a la población mexicana, de entre diez a cuarenta años; con el objetivo de analizar el uso e interacción con sus dispositivos electrónicos. Dentro de los rubros del cuestionario se encuentran el incremento del tiempo en línea y las modificaciones en las prácticas de

\section{Abstract}

During the pandemic that emerged at the root of the disease COVID-19, security measures were implemented that involved the confinement and distance of the population. This changed the lifestyles of individuals through Information and Communication Technologies (ICT). For the purposes of this investigation, we focus on social-digital networks (RSD), which are of significant importance for individual and organizational development. Therefore, we sought to contrast the use of various applications, before and during the pandemic by means of the probabilistic implementation of a questionnaire in the longitudinal to the Mexican settlement between ten and forty years with the aim of analyzing the use and interaction with its electronic devices. Within the rubles of the questionnaire there is an increase in time online, changes in sociability practices, acquisition and use of new applications and pages to shape a new 
sociabilidad, adquisición y uso de nuevas aplicaciones y páginas para la conformación de un nuevo estilo de vida. Debido a esto, se consideró enfocar este estudio no experimental transeccional a la descripción del consumo de RSD durante la pandemia. Los resultados expresan un aumento significativo en el uso del internet, así como cambios en cuanto a la utilización de ciertas aplicaciones, al respecto de las actividades que realizan en línea en su mayoría aumentaron. En conclusión, ha aumentado el uso del internet generando nuevos procesos de interacción social, convirtiéndose, así, en un estilo de vida o parte de la cotidianeidad.

Palabras clave: Medios sociales, internet, aplicación de telecomunicaciones, estilo de vida, pandemia, tecnologías de la información y la comunicación. lifestyle. Due to this, it was considered to focus this study on the experimental transcriptional description of RSD consumption during the pandemic. The results express a significant increase in the use of the internet, as well as changes in the use of certain applications regarding the activities that they carry out major increase of the use online. In conclusion, the use of the internet has increased, generating new processes of social interaction and converting into a lifestyle or part of everyday life.

Keywords: social media, internet, Telecommunications applications, lifestyle, pandemia, information technologies and communication.

\section{INTRODUCCIÓN}

A finales de 2019, China notificó la presencia de una nueva enfermedad cuya propagación fue acelerada y de gran alcance, esto eventualmente creó una pandemia global que alteraría la cotidianeidad a la que el mundo estaba habituado. Dicha enfermedad sería nombrada como COVID-19, considerada en el 2020 por la Organización Mundial de la Salud (OMS) como una amenaza a la salud (OMS, 2020). En un intento por controlar su contagio, los gobiernos han tomado políticas principalmente orientadas al distanciamiento social y al confinamiento que han transformado los entornos sociales (Comisión Económica para América Latina y el Caribe (CEPAL), 2020). No obstante, esta medida ha afectado las actividades educativas, comerciales, turísticas, laborales, sanitarias y cualquiera que implique contacto físico entre las personas, debido a que se ha requerido la adaptación de las mismas por medio de la digitalización en favor de evitar exponer a las personas ante el virus.

Por ello, el confinamiento en México fue de carácter obligatorio, lo que dejó a la población aislada entre sí por amplios periodos de tiempo y derivó en el incremento del uso de las Tecnologías de la información y comunicación (TIC) como una herramienta para realizar las actividades cotidianas.

En este trabajo se ha planteado que la relevancia actual de las redes sociodigitales (RSD) se debe a causa de las circunstancias vividas durante la pandemia y que el incremento en su uso ha repercutido en los hábitos de sociabilidad, modificando los estilos de vida. Por ello, se ha realizado una investigación con el fin de identificar las transformaciones dadas en el uso de las TIC a través del consumo de bienes y servicios por parte de los mexicanos durante la pandemia, así como el estudio de configuración de estilo de vida que dichas personas han tenido a partir de las prácticas del confinamiento. Para llevar a cabo lo anterior, se realizó una muestra no probabilística que consistió en desarrollar y aplicar trescientos cuatro 
cuestionarios digitalmente a sujetos entre diez a cuarenta años, durante los meses de junio a agosto del año 2020.

\subsection{Tecnologías de la información y comunicación y Redes sociodigitales}

Las TIC se han configurado como los medios preferidos de la población para informarse y entretenerse; fenómeno que se agudizó con la pandemia y ha causado una mayor dependencia de los seres humanos hacia los dispositivos tecnológicos afectando y modificando los hábitos cotidianos y los estilos de vida. Así, las TIC juegan un papel esencial en la vida del ser humano. Según Cobo Román, estas son:

Dispositivos tecnológicos (hardware y software) que permiten editar, producir, almacenar, intercambiar y transmitir datos entre diferentes sistemas de información que cuentan con protocolos comunes. Estas aplicaciones, que integran medios de informática, telecomunicaciones y redes, posibilitan tanto la comunicación y colaboración interpersonal (persona a persona) como la multidireccional (uno a muchos o muchos a muchos). Estas herramientas desempeñan un papel sustantivo en la generación, intercambio, difusión, gestión y acceso al conocimiento. (2009, p. 312)

Las TIC ofrecen una variedad de beneficios para el ser humano, facilitando la interacción entre personas; no obstante, también pueden ser perjudiciales para los mismos. Por lo que es necesario entrar en detalle sobre cómo estas impactan a la sociedad. Las ventajas de las TIC son: acceso a las fuentes de información, automatización de los trabajos, canales de comunicación inmediata conjuntas a dinámicas de intercambio de información, capacidad de almacenamiento, interactividad, interdisciplinariedad, procesamiento rápido de los datos y la capacidad de manejar la información, ya sea, textual, audiovisual, multimedia e hipertexto (Ávila, 2003; López y Villafañe 2011). De esta forma, se observa que los beneficios en muchos ámbitos de la vida humana han sido vastos, además, en la época de la pandemia, las TIC han desplegado múltiples posibilidades de continuar con la educación, empleos, compras y adquisición de servicios, entre otros, desde casa.

Por otro lado, se encuentran ciertas desventajas como lo son las barreras económicas para adquirir dispositivos; la posible homogeneidad cultural; procesos educativos y sociales poco humanos; problemas de seguridad y el desinterés de las personas a adaptarse a su uso (Ávila, 2003; Sánchez, 2008; López y Villafañe, 2011).

Dentro de las TIC se encuentran las redes sociales o medios sociales (social media). Para distinguirlas de la teoría tradicional sociológica de las redes sociales (Criado y Rojas, 2015). En el presente trabajo se usará el término de redes sociodigitales (RSD), las cuales se comprenden como "el enjambre de la comunicación digital" (Winocur y Sánchez, 2015, p. 9). De esta manera, la expresión redes sociodigitales implica la codificación en formato digital (Incera, Cartas y Cairo, 2007) y permite abordar el fenómeno de forma operativa e inclusiva ya que abarca la diversidad de aplicaciones y plataformas de servicios orientados a lo social (Conti y Pasarella, 2017).

En investigaciones previas acerca de las RSD se encontró que dichas redes están impregnadas de las actitudes humanas, lo que las conduce a ser consideradas como esenciales. Por otra parte, también apoyan en la generación de puentes de comunicación entre estudiantes, profesores y otros individuos (Cortés, et al., 2015). Así, sirven como una "herramienta para el 
activismo, principalmente de las organizaciones no gubernamentales (ONGs), ciudadanos, medios de comunicación, políticos y activistas que han aprovechado la interactividad e inmediatez del medio para hacer escuchar sus voces" (Gómez y Sosa-Plata, 2012).

Las RSD permiten a los usuarios, según la naturaleza del sitio: a) construir un perfil público o semipúblico dentro de un sistema delimitado, b) que se comuniquen con otros usuarios, y c) relacionarse con las listas de contactos de otros usuarios en el sistema (Boyd y Ellison, 2007). Además, Cortés et al. (2015) consideran que las RSD son entendidas como sistemas de relación donde las personas entablan comunicación con contactos mediante plataformas asociadas al internet que posibilitan nuevos patrones de interacción social influenciada (Torres, 2013), para lo cual cada internauta las utiliza de manera diferente.

Además, el uso de las RSD tiene las ventajas de acceso a información y servicios para todos en cualquier momento y lugar desde una gran variedad de dispositivos (Incera, Cartas y Cairo, 2007). Con esto queda claro que las RSD permiten al individuo ser un sujeto vivo en lo virtual ya que se puede reflejar a sí mismo. No solamente se trata de estar dentro del mundo digital, sino que es más que espectador pasivo, donde cada usuario selecciona, interpreta, comprende y disfruta los contenidos que se encuentran en el mundo digital de comunicación, convirtiéndose así en "información, estructura y narrativa" (Hidalgo, 2014).

\section{Usos de TIC y RSD EN MÉxICO}

En México, el consumo del internet aún no se encuentra accesible a toda la población, por lo cual existe una brecha digital. No obstante, en este apartado se esbozará el acceso y usos que se tienen sobre las TIC en México basándonos en investigaciones revisadas. Dentro de ellos, se encontró que los grupos que más usan el internet son de 25 a 34 años con 20.2\% (hombres $10.4 \%$ y mujeres $9.8 \%$ ) y 18 a 24 años con $17.4 \%$ (mujeres $8.9 \%$ y hombres $8.6 \%$ ) (Instituto Federal de Telecomunicaciones et al., 2019). De manera similar, en el $14^{\circ}$ Estudio sobre los Hábitos de los Usuarios de Internet en México (Asociación de Internet, 2018) se halló que los jóvenes de 18 a 24 años representan el 17\% y entre las edades de 25 a 34 años el 19\% son internautas, así, ambos representan el 36\% de los internautas (Asociación de Internet, 2018). De forma idéntica, el estudio de Encuesta Nacional de Consumo Cultural de México (ENCCUM) observó que la población entre 18 y 29 años fue quien más usa Internet por motivos culturales, esto con el equivalente a un tercio del total de la población (INEGI, 2012).

Por lo general, se comprende que las TIC requieren de dispositivos que permitan su acceso. Para ello, tenemos que saber a través de qué dispositivos se da el uso de las RSD en la población mexicana. Estos son: celular inteligente, computadora portátil, computadora de escritorio, tableta, videojuegos, aparatos electrónicos, servicios móviles portátiles de mano y otros dispositivos (Asociación de Internet, 2019; Instituto Federal de Telecomunicaciones et al., 2019; Ochoa-Ontiveros, 2019.

En cuanto a las redes más usadas por la generación Z, se prefirieron aquellas de interacción con terceros y las que predominaron fueron: Facebook, WhatsApp, YouTube, Instagram, Twitter, Snapchat, Pinterest, Google+, Tumblr y WeHeartilt (IAB México y Televisa digital, 2018). Lo cual concuerda con las actividades que prefieren los usuarios que son la conexión con sus seres queridos, así como con las personas de su generación o profesión, además de compartir intereses, inquietudes y motivaciones con diversas personas. 
En Similarweb y Comscore (2020), páginas de ranking revisadas durante noviembre del 2020 se encontró que entre las aplicaciones más utilizadas a lo largo de ese mes están WhatsApp, Google, Facebook, YouTube, Instagram, Twitter, TikTok, Spotify, Netflix y Disney+. También se constató según el $14^{\circ}$ Estudio sobre los Hábitos de los Usuarios de Internet en México que la red social favorita de los mexicanos es Facebook, de la cual 98\% de internautas tiene conocimiento, WhatsApp es la segunda con $91 \%$, YouTube la tercera con $82 \%$, sigue Instagram con $57 \%$ y Twitter con $49 \%$ (2018). Aunado a todo lo anterior, los usuarios pasan $40 \%$ de su tiempo conectados al internet en alguna red social y el $64 \%$ de los internautas están conectados durante todo el día en actividades en línea, lo cual desencadenó que un 68\% tuviera un cambio en sus hábitos debido al uso del internet (Asociación de Internet, 2018).

Las TIC son parte esencial de nuestras vidas porque a través de diferentes dispositivos podemos hacer uso de las RSD, permitiéndonos, en tanto usuarios, construir un perfil para tomar posición en el espacio sociodigital, lo cual determina nuestro papel social. Entonces, las RSD pueden consolidar un sistema de prácticas en diferentes plataformas que integran las experiencias pasadas y funcionan como una matriz de interpretación acorde a las percepciones que derivan en acciones futuras, donde usualmente hay una constante reiteración de esta, por la actuación que se determina por su posición en el espacio social; habilitando y deshabilitando disposiciones y convirtiéndose en lo que Bourdieu entiende como habitus (Bourdieu, 1991; Capdevielle, 2011).

Los habitus, de acuerdo a Bourdieu (1991) son procesos sociales inculcados e incorporados. Estos se hallan configuran o modifican en función de la nula, baja o alta integración, por medio de la acción e interpretación emocional y lógica (Castaño, 2011). De esa manera, podemos afirmar que el habitus está influenciado por los cambios culturales, se manifiestan tanto de manera individual como social, por lo que son capaces de transformar los modelos compartidos por un grupo (Guerra, 2010).

Para fines de esta investigación se define al consumo como la adquisición de productos o servicios. Con la expresión hábitos de consumo entenderemos pues, a todas aquellas prácticas o disposiciones de actos repetidos para adquisición de bienes y servicios. Los hábitos de consumo han existido a lo largo de la humanidad y son diferenciados a través de estudios generacionales (Tavera, 2018), estos se van constituyendo como estilos de vidas basado en aspiraciones, deseos, necesidades y cambios sociales, es entonces que el entorno y la socialización influyen en sus formas de percibir y maniobrar prácticas individuales/colectivas; por lo cual esta expresión se entiende como una construcción social.

Por medio de los habitus, se consolidan los comportamientos, actitudes, costumbres, tradiciones, actividades y decisiones (Sanabria-Ferrand., et al, 2007). Cuando estos se vuelven cotidianos en una persona (Arrivillaga, Salazar, Correa, 2003), ya sea para llevar comportamientos positivos como riesgosos para su vida (Palomares,2014), a todo esto, se le llama estilo de vida. La OMS define lo anterior como la forma de vida mediada por los factores socioculturales y las características personales que disminuyen el riesgo de enfermarse o morir a temprana edad. El individuo, al adquirir estilos de vida saludable, mejorará su salud y disfrutará de otros aspectos (Health Education Unit, 1986). Al adoptar estos comportamientos saludables, se mantiene el bienestar, la calidad de vida, la satisfacción de las necesidades propias y del desarrollo humano (Palomares, 2014). 
Además, Pullen, Noble y Fland (2001), afirmaron que estos son producto de las dimensiones personales, ambientales y sociales que rodean al individuo, tanto de su presente como de su historia; pudiéndose afirmar que es un proceso de aprendizaje por asimilación o imitación de patrones familiares o grupos formales e informales (Sanabria-Ferrand., et al, 2007). Todas las definiciones coinciden en que el estilo de vida es un conjunto de comportamientos socioculturales que tienen repercusiones en la salud psicológica como física de las personas. El estilo de vida es resultado de la interacción del contexto ambiental, genético, económico, educativo, familiar, entre otros; que tienen un peso en la construcción de cierto comportamiento a través de actitudes y valores que expresan el comportamiento del individuo en las diferentes áreas de la vida como la actividad física, la alimentación, el manejo de la sexualidad y las condiciones de vida como el ambiente social, físico, cultural y los diferentes aspectos económicos de la vida de las personas (Guerrero y León, 2010), agregamos que las TIC también tienen influencia en los diferentes factores del estilo de vida ya que ayudan a informarse en varios temas sociales que fomentan una mejor calidad de vida en las personas.

\section{Metodología}

El trabajo se realizó según las siguientes cuatro etapas: 1) diseño de la encuesta 2) aplicación de la encuesta a través de formularios Google 3) procesamiento de la información con SPSS y matriz de contenido 4) descripción de resultados (Zapata, 2005). Para lo anterior, se llevó a cabo un diseño de investigación (no experimental) transeccional descriptivo. Esto implica la indagación de las variables descriptivas en una determinada población, recolectando datos en un momento dado (Hernández, Fernández y Baptista, 2014). Esto se realizó a través de un cuestionario auto aplicado en internet por un formulario de Google durante los meses de junio a agosto del 2020, con un total de 327 respuestas; para lo cual la delimitación de los sujetos tuvo en cuenta las siguientes características: tener de diez a cuarenta años, género indistinto, que radicaran en el país de México y se pidió que proporcionaran su correo electrónico como consentimiento. La muestra fue no probabilística y orientada a la investigación cualitativa, es decir que el tamaño de la muestra no se seleccionó aleatoriamente, sino que fue por conveniencia y se buscó que estos fueran de máxima variación (Hernández, Fernández y Baptista, 2014). Sin embargo, la muestra seleccionada no pretende proveer los datos representativos de toda la población.

De los cuestionarios aplicados solamente 304 fueron validados, de la población encuestada el $56.6 \%$ está representada por el sexo femenino asignado al nacer y el $43.4 \%$ por el sexo masculino. Los tópicos abordados en las preguntas fueron sociodemográficos y panorámica general de sus usos del internet, ya sean dispositivos, RSD, tiempos dedicados y sociabilidad en estos tanto antes como durante el COVID-19 para comparar los datos descriptivos.

Durante el cuestionario se aplicó un total de ciento ochenta y dos preguntas, en la parte sociodemográfica fueron diecisiete preguntas cerradas y una abierta. En la panorámica general fueron once preguntas abiertas y ciento cincuenta y tres cerradas; de las preguntas cerradas del cuestionario en su mayoría fueron de opción múltiple y el resto dicotómicas. Para el presente artículo se incluyeron los resultados de setenta preguntas cerradas y siete de las abiertas, para el análisis de la información se usó el programa SPSS en las cerradas. Para las preguntas abiertas, se diseñó un sistema de jerarquización por frecuencia de repetición. Para 
el presente artículo únicamente se anexan cuatro gráficos y una tabla, no obstante, se mencionan las diferencias más importantes encontradas en la información obtenida.

\section{INTERPRETACIÓN Y DISCUSIÓN DE RESULTADOS}

Para el propósito de este trabajo se realizó una comparación entre antes y después de la pandemia del COVID-19, para corroborar si efectivamente nos encontrábamos ante un incremento en el consumo de RSD. De esta forma, los siguientes resultados serán, en su mayoría, una comparativa entre las dos temporalidades.

El 91.1\% dice contar con internet móvil en su casa y respecto a cómo se conectan al internet: el $64.5 \%$ con wifi, el $27.3 \%$ con ambos, el $7.6 \%$ con datos, $0.3 \%$ internet del vecino y $0.3 \%$ ninguno de los anteriores. En cuanto a la utilización de dispositivos de internet, respondieron que tenían los siguientes: doscientos y unas personas poseían computadoras de escritorio, el mismo número de personas contaban con televisión, ciento noventa personas con ordenadores portátiles, ochenta y un personas tabletas, sesenta y un personas celulares y diecinueve personas relojes inteligentes. No obstante, las personas que no cuentan con algunos de los dispositivos anteriores mencionaron utilizar un smartphone u otro dispositivo inteligente para conectarse a internet (38.51\%).

Por otro lado, se preguntó a los participantes cuántas horas dedicaban a la utilización de computadoras, laptops, smartphones o tabletas, así como el consumo de internet diario. Los resultados, como se evidencia en la tabla 1 , demuestran un aumento en cuanto al antes y durante la pandemia en ambos casos. Así, observamos un incremento en el uso de dispositivos y del consumo de internet diario, debido a que los nuevos estilos de vida implican un mayor uso de las RSD y TIC.

Tabla 1

El uso de horas en los dispositivos y el internet antes y durante la pandemia

\begin{tabular}{|c|c|c|c|c|}
\hline & \multicolumn{2}{|c|}{ Uso de dispositivos } & \multicolumn{2}{c|}{ Uso del internet } \\
\hline & Antes & Durante & Antes & Durante \\
\hline Media & 5.758 & 8.52 & 6.081 & 9.74 \\
\hline Mediana & 5 & 8 & 5 & 9 \\
\hline Moda & 4 & 8 & 4 & 12 \\
\hline Mínimo & 0 & 0 & 0 & 0 \\
\hline Máximo & 100 & 24 & 24 & 28 \\
\hline
\end{tabular}

Fuente: elaboración propia.

Antes del COVID-19, la intención con la cual los encuestados ingresaban a los sitios web o a las RSD fue principalmente para buscar información: en general (17.83\%), académica (14.62\%), sobre salud (8.52\%), eventos de interés (16.52\%) y búsqueda de empleo (6.42\%). Sobre las relaciones sociales: hacer amistades (28.77\%), encontrar con quien tener sexo (3.80\%) y por último encontrar pareja (3.44\%). Durante el COVID-19 ingresaban para búsqueda de información miscelánea (18.79\%), académica (16.59\%), sobre salud (15.61\%), eventos (12.30\%) y búsqueda de empleo (4.94\%). En cuanto a relaciones interpersonales respondieron: hacer amistades (26.70\%), encontrar con quien tener relaciones sexuales (3.18\%) y encontrar 
pareja (2.85\%). Podemos ver que hubo una disminución en la búsqueda de fomentar las relaciones interpersonales (hacer amistades, relaciones sexuales y encontrar pareja). En contraste, aumentó la búsqueda de información en general, académica y salud. La búsqueda de empleo decayó durante la aplicación de este cuestionario, suponemos que por los estragos económicos que existirán durante la pandemia habrá pérdida de empleos y por lo tanto este resultado habrá de cambiar a mayor búsqueda de empleos.

En cuanto al uso de RSD, se encontró que las personas tenían instaladas, previo al cuestionario, aplicaciones para: mensajería instantánea (WhatsApp, Messenger, Telegram, etc.) en un $18.90 \%$ de las veces, las redes sociales (Facebook, Instagram, Twitter, etc.) obtuvieron un $14.63 \%$, los contenidos de audio y video (YouTube, Spotify, Deezer, etc.) un 13.92\%, el contenido educativo y de formación (Khan Academy, Kahoot, Canva etc.) fue de 9.65\%, bienes y servicios (Cabify, Mercadolibre, Rappi, Uber, etc.) con $8.86 \%$, la banca móvil (Bancomer, Banamex, etc.) con 8.30\%, para el aprendizaje de idiomas (Duolingo, etc.) $7.83 \%$, los juegos (Pokemon Go, Candy Crush, Among Us, etc.) consiguieron un 7.35\% y la utilización de servicios de alojamiento y viajes (Booking, Airbnb, Expedia, etc.) un $3 \%$.

En la tabla 2 se observa que las aplicaciones más comunes eran y siguen siendo WhatsApp, Facebook e Instagram. Por consiguiente, observamos que previo a la pandemia predominaban las aplicaciones de sociabilidad y en décimo segunda posición figuraba una aplicación académica, en contraste durante esta aparecieron aplicaciones que previo a la pandemia no figuraban y la única académica que aparecía subió al número cinco. De forma idéntica a las aplicaciones, las páginas web que más usan y siguen usando encontramos Google, Youtube y Facebook. De este modo en las páginas web consumían principalmente las redes de búsqueda, entretenimiento, sociabilidad y compras, ahora figuran entre las principales, algunas que sirven para lo académico e incluso figuran otras páginas de entretenimiento.

Tabla 2

Las aplicaciones y páginas web usadas antes y durante la pandemia

\begin{tabular}{|c|c|c|c|c|c|c|c|c|c|c|c|}
\hline \multicolumn{3}{|c|}{ Aplicaciones durante } & \multicolumn{3}{|c|}{ Aplicaciones antes } & \multicolumn{3}{|c|}{ Págs. Web durante } & \multicolumn{3}{|c|}{ Págs. Web antes } \\
\hline & Frec. & $\%$ & & Frec. & $\%$ & & Frec. & $\%$ & & Frec. & $\%$ \\
\hline WhatsApp & 237 & 20.39 & WhatsApp & 263 & 22.71 & Google & 96 & 15.97 & Google & 91 & 17.36 \\
\hline Facebook & 185 & 15.92 & Facebook & 245 & 21.15 & YouTube & 70 & 11.64 & Facebook & 80 & 15.26 \\
\hline Instagram & 120 & 10.32 & Instagram & 171 & 14.76 & Facebook & 60 & 9.98 & YouTube & 72 & 13.74 \\
\hline YouTube & 88 & 7.57 & YouTube & 122 & 10.53 & WhatsApp & 49 & 8.15 & WhatsApp & 46 & 8.77 \\
\hline Classroom & 70 & 6.02 & Messenger & 89 & 7.68 & Classroom & 43 & 7.15 & Instagram & 32 & 6.10 \\
\hline Zoom & 55 & 4.73 & Twitter & 61 & 5.26 & Correo & 42 & 6.98 & Correo & 32 & 6.10 \\
\hline Messenger & 47 & 4.04 & Snapchat & 39 & 3.36 & Wikipedia & 32 & 5.32 & Wikipedia & 30 & 5.72 \\
\hline Twitter & 45 & 3.87 & Netflix & 27 & 2.33 & Netflix & 20 & 3.32 & Netflix & 19 & 3.62 \\
\hline Tik Tok & 41 & 3.52 & Spotify & 23 & 1.98 & Zoom & 19 & 3.16 & Messenger & 16 & 3.05 \\
\hline Netflix & 37 & 3.18 & Google & 22 & 1.89 & MercadoLibre & 12 & 1.99 & Twitter & 14 & 2.67 \\
\hline Google & 35 & 3.01 & Tik Tok & 17 & 1.46 & Canva & 12 & 1.99 & MercadoLibre & 11 & 2.09 \\
\hline Correo & 34 & 2.92 & Classroom & 9 & 0.77 & Moodle & 11 & 1.83 & Amazon & 10 & 1.90 \\
\hline
\end{tabular}




\begin{tabular}{|l|l|l|c|c|c|c|c|c|c|c|c|}
\hline Spotify & 25 & 2.15 & Uber & 8 & 0.69 & Xvideos & 9 & 1.49 & Spotify & 9 & 1.71 \\
\hline Meet & 24 & 2.06 & Correo & 6 & 0.51 & Amazon & 8 & 1.33 & Classroom & 6 & 1.14 \\
\hline Snapchat & 19 & 1.63 & Duolingo & 6 & 0.51 & Tik Tok & 7 & 1.16 & Pinterest & 5 & 0.95 \\
\hline
\end{tabular}

Fuente: elaboración propia.

El entretenimiento en internet ha tenido un aumento significativo en la pandemia, siendo este una de las principales formas de ocio. Sobre esta forma de entretenimiento, la población encuestada mencionó que: rara vez revisaban información en línea acerca de eventos o pasatiempos, durante el COVID-19 esto aumentó. Les preguntamos a los usuarios sobre la frecuencia de utilización del internet en diversos dispositivos y los datos arrojan que el uso del internet en consolas de videojuegos vio un incremento de rara vez a frecuente. Acerca del uso en los reproductores multimedia declararon que de poco frecuente el consumo aumentó a muy frecuente. En cuanto a la búsqueda de pornografía, se encontró que predomina el "nunca". Tanto antes como después de la pandemia, el uso de internet para escuchar música permaneció en "frecuente", y ver películas y programas televisivos fue poco frecuente, en tanto, durante la pandemia se reportó como habitual. Además, en la lectura de revistas de ocio y periódicos predominó el casi nulamente siendo ahora regular. Relacionado a lo anterior, la búsqueda de información y documentación escolar/laboral era frecuente y se mantuvo de este modo con un ligero aumento, véase tabla 3 para más datos.

Respecto al entretenimiento no todo es siempre en línea, sino que en muchos casos los cibernautas descargan la información de su interés para consumirla aún sin conexión. En las descargas: de música y de lectura de textos y libros se observa que eran pocas, a diferencia de en la pandemia cuando se volvió frecuente; de videos y películas antes del COVID-19 era prácticamente nula, en contraste de hoy en día que es regular y de juegos antes de la pandemia rara vez y esto se mantuvo similar durante la pandemia salvo por un ligero aumento (Véase tabla 3).

Tabla 3

El uso del internet mediante dispositivos o para las siguientes actividades de entretenimiento tanto antes como durante la pandemia

\begin{tabular}{|c|c|c|c|c|c|c|c|c|}
\hline & \multicolumn{4}{|c|}{ Antes } & \multicolumn{4}{|c|}{ Durante } \\
\hline & Nunca & Poco & $\begin{array}{l}\text { Frecuent } \\
\text { e }\end{array}$ & Siempre & Nunca & Poco & $\begin{array}{l}\text { Frecuent } \\
\mathrm{e}\end{array}$ & $\begin{array}{l}\text { Siempr } \\
\text { e }\end{array}$ \\
\hline $\begin{array}{l}\text { En consolas de } \\
\text { videojuegos }\end{array}$ & $\begin{array}{l}35.50 \\
\%\end{array}$ & $\begin{array}{l}40.80 \\
\%\end{array}$ & $17.80 \%$ & $5.90 \%$ & $\begin{array}{l}23.70 \\
\%\end{array}$ & $\begin{array}{l}20.40 \\
\%\end{array}$ & $30.90 \%$ & $25 \%$ \\
\hline $\begin{array}{l}\text { En los reproductores } \\
\text { multimedia }\end{array}$ & $5.90 \%$ & $27 \%$ & $42.80 \%$ & $24.30 \%$ & $6.30 \%$ & $\begin{array}{l}16.10 \\
\%\end{array}$ & $39.50 \%$ & $38.20 \%$ \\
\hline Búsqueda de pornografía & $\begin{array}{l}66.10 \\
\% \\
\end{array}$ & $\begin{array}{l}18.10 \\
\%\end{array}$ & $11.50 \%$ & $4.30 \%$ & $\begin{array}{l}54.30 \\
\% \\
\end{array}$ & $\begin{array}{l}12.20 \\
\%\end{array}$ & $17.80 \%$ & $15.80 \%$ \\
\hline Para escuchar música & $7.60 \%$ & $\begin{array}{l}33.20 \\
\%\end{array}$ & $35.50 \%$ & $23.70 \%$ & $7.60 \%$ & $\begin{array}{l}24.30 \\
\%\end{array}$ & $37.50 \%$ & $30.60 \%$ \\
\hline $\begin{array}{l}\text { Ver películas y } \\
\text { programas televisivos }\end{array}$ & $\begin{array}{l}11.50 \\
\%\end{array}$ & $\begin{array}{l}30.90 \\
\%\end{array}$ & $35.90 \%$ & $21.70 \%$ & $8.60 \%$ & $\begin{array}{l}20.40 \\
\%\end{array}$ & $40.50 \%$ & $30.60 \%$ \\
\hline $\begin{array}{l}\text { Leer revistas de ocio y } \\
\text { periódicos }\end{array}$ & $\begin{array}{l}42.40 \\
\%\end{array}$ & $\begin{array}{l}37.20 \\
\%\end{array}$ & $16.40 \%$ & $3.90 \%$ & $\begin{array}{l}28.30 \\
\%\end{array}$ & $\begin{array}{l}27.60 \\
\%\end{array}$ & $31.30 \%$ & $12.80 \%$ \\
\hline $\begin{array}{l}\text { revisar información } \\
\text { sobre eventos o } \\
\text { pasatiempos }\end{array}$ & $\begin{array}{l}29.90 \\
\% \\
\end{array}$ & $\begin{array}{l}37.20 \\
\%\end{array}$ & $26.30 \%$ & $6.60 \%$ & $\begin{array}{l}21.70 \\
\%\end{array}$ & $\begin{array}{l}33.20 \\
\%\end{array}$ & $30.60 \%$ & $14.50 \%$ \\
\hline Búsqueda de & 15.50 & 30.30 & $35.20 \%$ & $19.10 \%$ & 15.80 & 18.40 & $39.50 \%$ & $26.30 \%$ \\
\hline
\end{tabular}




\begin{tabular}{|l|l|l|l|l|l|l|l|l|}
\hline $\begin{array}{l}\text { información y } \\
\text { documentación } \\
\text { escolar/laboral }\end{array}$ & $\%$ & $\%$ & & $\%$ & $\%$ & & \\
\hline $\begin{array}{l}\text { Descargas de textos y } \\
\text { libros }\end{array}$ & $\begin{array}{l}26.30 \\
\%\end{array}$ & $\begin{array}{l}37.80 \\
\%\end{array}$ & $27.60 \%$ & $8.20 \%$ & $\begin{array}{l}19.40 \\
\%\end{array}$ & $\begin{array}{l}30.60 \\
\%\end{array}$ & $31.30 \%$ & $18.80 \%$ \\
\hline Descargas de música & $\begin{array}{l}18.10 \\
\%\end{array}$ & $\begin{array}{l}36.50 \\
\%\end{array}$ & $30.30 \%$ & $15.10 \%$ & $\begin{array}{l}19.10 \\
\%\end{array}$ & $\begin{array}{l}24.30 \\
\%\end{array}$ & $34.20 \%$ & $22.40 \%$ \\
\hline $\begin{array}{l}\text { Descarga de videos y } \\
\text { películas }\end{array}$ & $\begin{array}{l}30.60 \\
\%\end{array}$ & $\begin{array}{l}37.20 \\
\%\end{array}$ & $21.10 \%$ & $11.20 \%$ & $\begin{array}{l}22.70 \\
\%\end{array}$ & $\begin{array}{l}23.40 \\
\%\end{array}$ & $33.20 \%$ & $20.70 \%$ \\
\hline Descarga de juegos & $\begin{array}{l}39.10 \\
\%\end{array}$ & $\begin{array}{l}40.10 \\
\%\end{array}$ & $12.20 \%$ & $8.60 \%$ & $\begin{array}{l}28.30 \\
\%\end{array}$ & $\begin{array}{l}26.30 \\
\%\end{array}$ & $28.60 \%$ & $16.80 \%$ \\
\hline
\end{tabular}

Fuente: elaboración propia.

Como las personas se encuentran en un aislamiento que los ha llevado a buscar otras formas de relacionarse constantemente, ya sean familia, amigos, compañeros u otras personas, han encontrado en el acto de compartir un sustituto al contacto presencial. En la subida de fotos o videos personales con familia, amigos y compañeros se mantienen las mismas categorías predominantes poco frecuente con un ligero aumento en frecuente. Además, los usuarios mencionaron que escasamente compartían archivos antes y durante la pandemia. En cuanto a compartir o reenviar información de otras personas y al intercambiar información y opiniones con otras personas antes de la pandemia los participantes reportaron hacerlo rara vez y esto aumentó durante la misma. También, los usuarios dijeron que nunca mantenían un sitio web o blog propio y en su mayoría siguen sin hacerlo. Asimismo, seguir blogs o webs fue poco y se mantuvo en rara vez durante la pandemia. En contraste, la muestra reportó que, al observar información de amistades, familiares o personas cercanas, otras personas, personas desconocidas o que casi no conocen, hubo un aumento durante la pandemia, así como los participantes reportaron que era poco el contacto que mantenían con personas que no podían ver de manera física (v. tabla 4).

El consumo forma parte de nuestra sociedad ya que este nos significa y posiciona socialmente a través de la comunicación. En cuanto a este en las RSD, los encuestados mencionaron que fue bastante frecuente y se mantuvo la frecuencia durante la pandemia. Las videollamadas o llamadas eran poco regulares y ahora son bastante regulares. Respecto a la mensajería instantánea (WhatsApp, Line, Telegram, etc.), anteriormente se encontró que muy frecuentemente la usaban y se mantuvo. El correo electrónico era poco frecuente y tuvo un aumento a muy frecuente. El crear los grupos con correos o chats de compañeros previamente era poco usual, no obstante, se da de manera muy frecuente en la pandemia, véase la tabla 4.

Tabla 4

El uso del internet en las siguientes actividades de comunicación tanto antes como durante la pandemia

\begin{tabular}{|l|c|c|c|c|c|c|c|c|}
\hline & \multicolumn{4}{|c|}{ Antes } & \multicolumn{4}{c|}{ Durante } \\
\hline & Nunca & Poco & Frecuente & Siempre & Nunca & Poco & Frecuente & Siempre \\
\hline $\begin{array}{l}\text { Las videollamadas o } \\
\text { Ilamadas }\end{array}$ & $15.80 \%$ & $43.80 \%$ & $27.30 \%$ & $13.20 \%$ & $12.50 \%$ & $19.10 \%$ & $37.50 \%$ & $30.90 \%$ \\
\hline Mensajería instantánea & $5.90 \%$ & $21.10 \%$ & $28.90 \%$ & $44.10 \%$ & $6.90 \%$ & $12.80 \%$ & $32.20 \%$ & $48 \%$ \\
\hline $\begin{array}{l}\text { RSD (Facebook, } \\
\text { Instagram, Twitter, } \\
\begin{array}{l}\text { Wattpad, Snapchat, } \\
\text { YouTube, etc.) }\end{array}\end{array}$ & $5.90 \%$ & $20.70 \%$ & $34.20 \%$ & $39.10 \%$ & $7.60 \%$ & $14.10 \%$ & $32.60 \%$ & $45.70 \%$ \\
\hline Correo electrónico & $15.50 \%$ & $30.30 \%$ & $32.60 \%$ & $21.70 \%$ & $9.90 \%$ & $16.40 \%$ & $42.10 \%$ & $31.60 \%$ \\
\hline
\end{tabular}


Transformaciones en los hábitos de comunicación y sociabilidad a través del incremento del uso de redes sociodigitales en tiempos de pandemia

Rubén Cervantes Hernández / Dra. Paola Margarita Chaparro-Medina

\begin{tabular}{|c|c|c|c|c|c|c|c|c|}
\hline $\begin{array}{l}\text { Crear los grupos con } \\
\text { correos o chats de } \\
\text { compañeros }\end{array}$ & $24.00 \%$ & $39.80 \%$ & $24.00 \%$ & $12.20 \%$ & $18.40 \%$ & $22.40 \%$ & $33.90 \%$ & $25.30 \%$ \\
\hline $\begin{array}{l}\text { Subida de fotos o videos } \\
\text { personales con familia, } \\
\text { amigos y compañeros }\end{array}$ & $15.80 \%$ & $42.80 \%$ & $28.60 \%$ & $12.80 \%$ & $19.70 \%$ & $29.90 \%$ & $29.30 \%$ & $21.10 \%$ \\
\hline Compartir archivos & $45.70 \%$ & $34.50 \%$ & $13.20 \%$ & $6.60 \%$ & $29.60 \%$ & $28.30 \%$ & $26.30 \%$ & $15.80 \%$ \\
\hline $\begin{array}{l}\text { Compartir o reenviar } \\
\text { información de otras } \\
\text { personas }\end{array}$ & $35.20 \%$ & $38.50 \%$ & $20.10 \%$ & $6.30 \%$ & $29.60 \%$ & $25.70 \%$ & $29.30 \%$ & $15.50 \%$ \\
\hline $\begin{array}{l}\text { Intercambiar información } \\
\text { y opiniones con otras } \\
\text { personas }\end{array}$ & $135.20 \%$ & $38.50 \%$ & $20.10 \%$ & $6.30 \%$ & $22.40 \%$ & $28.00 \%$ & $30.60 \%$ & $19.10 \%$ \\
\hline $\begin{array}{l}\text { Mantener un sitio web o } \\
\text { blog propio }\end{array}$ & $67.10 \%$ & $21.10 \%$ & $8.60 \%$ & $3.30 \%$ & $50.70 \%$ & $18.80 \%$ & $16.80 \%$ & $13.80 \%$ \\
\hline $\begin{array}{l}\text { Participación activa en los } \\
\text { foros }\end{array}$ & $62.50 \%$ & $24.70 \%$ & $8.60 \%$ & $4.30 \%$ & $39.10 \%$ & $24.70 \%$ & $22.00 \%$ & $14.10 \%$ \\
\hline Seguir blogs o webs & $36.20 \%$ & $39.10 \%$ & $16.80 \%$ & $7.90 \%$ & $28.90 \%$ & $28.60 \%$ & $25.30 \%$ & $17.10 \%$ \\
\hline $\begin{array}{l}\text { Observar información de } \\
\text { amistades, familiares o } \\
\text { personas cercanas }\end{array}$ & $18.10 \%$ & $42.10 \%$ & $29.30 \%$ & $10.50 \%$ & $14.50 \%$ & $28.30 \%$ & $32.20 \%$ & $25.00 \%$ \\
\hline $\begin{array}{l}\text { Observar información } \\
\text { otras personas }\end{array}$ & $26.60 \%$ & $41.40 \%$ & $22.70 \%$ & $9.20 \%$ & $23.40 \%$ & $28 \%$ & $29.30 \%$ & $19.40 \%$ \\
\hline $\begin{array}{l}\text { Observar información de } \\
\text { personas desconocidas o } \\
\text { que casi no conocen }\end{array}$ & $41.80 \%$ & $36.50 \%$ & $17.10 \%$ & $4.60 \%$ & $28.30 \%$ & $28.30 \%$ & $25.70 \%$ & $17.80 \%$ \\
\hline $\begin{array}{l}\text { contacto con personas } \\
\text { que no ves } \\
\text { frecuentemente }\end{array}$ & $14.50 \%$ & $41.10 \%$ & $26.60 \%$ & $17.80 \%$ & $13.50 \%$ & $24.00 \%$ & $33.60 \%$ & $28.90 \%$ \\
\hline
\end{tabular}

Fuente: elaboración propia.

Por otra parte, en el internet de las cosas, el cual interconecta aparatos y monitoriza las actividades tecnológicas diarias del ser humano mediante diferentes dispositivos, la muestra reportó que tanto antes de COVID-19 como durante casi nunca emplean el internet de las cosas (Antes: nunca $46.40 \%$, poco $32.20 \%$, frecuente $16.80 \%$ y siempre $4.60 \%$. Durante: nunca $30.60 \%$, poco $28 \%$, frecuente $23.40 \%$ y siempre $18.10 \%$ ).

Entre otros datos, antes del COVID-19 el uso de WhatsApp u otra aplicación de mensajería instantánea se usaba, en su mayoría, para hablar con amistades (29.93\%) o familia (22.63\%) y para cuestiones relacionadas con los estudios y el trabajo (19.95\%), entre otros. Por otro lado, durante el COVID-19 predominaron las cuestiones relacionadas con los estudios y el trabajo (26.94\%), hablar con amistades (23.94\%) y, por último, hablar con la familia (20.28\%), entre otros. Aquí observamos que la percepción de los encuestados sobre su uso del internet se enfoca principalmente a lo educativo y laboral, quedando desplazadas sus relaciones sociales. Afirmamos que este cambio se debe al aislamiento porque obliga a las personas a tener gran parte de sus actividades en internet, sin embargo, eso no significa que sus formas de relacionarse por RSD hayan disminuido, sino que han cambiado sus estilos de vida.

En cuanto a la comunicación a través de sistemas de mensajería instantánea o mensajes de textos se encontró que: $67.4 \%$ tenía comunicación entre regular y constante con la familia y un $77.4 \%$ se comunica con sus amistades frecuentemente. De otra manera, la comunicación a 
través de redes sociales (Facebook, Instagram, Twitter, Wattpad, Snapchat, etc.) es: $55.5 \%$ tiene comunicación constante con su familia y $69.4 \%$ con sus amistades.

En estos últimos párrafos, se ha observado el papel de las RSD en la comunicación, la cual ha modificado la forma en que las personas se relacionan las unas con las otras. Sobre esto, se les preguntó a los encuestados sobre la manera en que ellos expresan más eficazmente sus sentimientos y respondieron lo siguiente: $36.8 \%$ prefiere hacerlo en persona y frente a los demás; $28.3 \%$ en persona y a solas; $9.2 \%$ en celular; $8.9 \%$ por RSD; $8.6 \%$ por mensajes de texto; $5.9 \%$ escribiéndolos en papel y $2.3 \%$ a través de letras de canciones. En cuanto los métodos que prefieren usar, la mayoría prefiere comunicarse en persona (67.4\%); otros tantos mensajear por internet (15.8\%). No obstante, solo un pequeño porcentaje gusta de hablar por internet (1.3\%), escribir correos electrónicos (1.3\%) y hablar por videollamada (0.7\%).

\section{CONCLUSIONES}

La situación mundial de la pandemia ha sido muy complicada para todos debido a que fue precipitada y sacudió los procesos sociales que se tenían. Gracias a las RSD se ha permitido la comunicación para poder efectuar varias actividades y tratar de continuar con las actividades diarias, nos hemos tenido que adaptar utilizando al internet como una herramienta que posibilita cumplir con las exigencias sanitarias y continuar en gran medida con la mayoría de las actividades que se realizaban previo a la pandemia. De este modo, hemos establecido que las TIC son todos los dispositivos que permiten crear, guardar, compartir y difundir contenido entre diferentes sistemas de información con el fin de posibilitar la comunicación y cooperación entre diversos entes individuales o colectivos/organizacionales y que las RSD, a su vez, quedan insertas dentro de las TIC, permitiendo una continua interacción entre sujetos individuales o grupales con diversos fines, ya sea adquirir servicios, productos, información, entretenimiento, entre otros.

Se planteó que había un incremento de las RSD por la pandemia y que estas modificaron algunos hábitos de sociabilidad lo cual se comprobó con el cuestionario aplicado al identificar usos y consumos que permitieron demostrar que hubo cambios en sus hábitos sociales debido a la pandemia COVID-19 (véase tablas 2-4). Se puede concluir que en los usos del internet se observan cambios significativos en el antes y durante la pandemia ya que se encuentra que en su uso de RSD las relaciones interpersonales se mantuvieron o en algunos casos cambiaron, debido a que usan ahora otras aplicaciones de trabajo o educativas (V. tablas 3-4). Además, las RSD permitieron a los ciudadanos y estudiantes dejar de ser pasivos en el ambiente digital y convertirse en sujetos un poco más interactivos en la conexión; eso no implica que antes no participaran, sino que hubo un ligero aumento en la participación de diferentes formas de estar inmersos en lo digital, ya sea por compartir fotos propias, archivos, reenviar información de otros, participar en páginas web e intercambiar información.

Hay un aumento de horas dedicadas al internet, no obstante, hay una aparente disminución de algunas aplicaciones, lo cual posiblemente se debe a que durante la pandemia figuran aplicaciones académicas y laborales entre las usadas, asimismo aparecen otras aplicaciones de sociabilidad que no figuraban antes del COVID-19. Las de mayor uso antes y durante son WhatsApp, Facebook e Instagram (V. tabla 2). Aseveramos que es un mero cambio del uso de unas aplicaciones por otras, ya que al observar las actividades en línea, en su mayoría tuvieron aumento del uso durante la pandemia (v. tabla 3 y 4 ). 
Con los resultados propios, podemos deducir que los hábitos están cambiando, que se están modificando los estilos de vida de las personas y que las TIC en la pandemia han jugado un papel esencial. Por ejemplo, la teleeducación que se está dando en escuelas públicas de México complementada con diversos chats de mensajería considerados RSD, a diferencia de las escuelas privadas mexicanas que utilizan las plataformas virtuales o RSD (Zoom y Skype) para impartir sus clases complementadas de igual manera con chats de mensajería. Además, en la tabla dos vemos que hay un incremento de las aplicaciones que podrían considerarse académicas.

Los datos recopilados son de carácter novedoso y su utilidad yace en el análisis del consumo durante la pandemia, que puede diferir bastante a todos los estudios previos. Al analizar esta información, se encontró que el consumo ha aumentado y, por ende, los hábitos de las personas están cambiando. Las posibles limitaciones de este estudio son la característica transversal en lugar de un enfoque longitudinal, así como que la muestra fue por conveniencia y no representativa. Por ello, sería relevante llevar a cabo investigaciones que permitan sondear sobre los efectos socioeconómicos, hábitos de consumo y sociabilidad durante y post pandemia. Además, de crear cuestionarios cortos con una muestra cuantitativa para generalizar los resultados. También aconsejamos tener en cuenta edades muy específicas para encontrar sus consumos en las RSD ya que debido a la amplitud del estudio propio consideramos que puede haber una variación muy extensa.

Para finalizar, los datos en el aumento de uso de las RSD refuerzan que la población ha tenido que adaptar sus hábitos a la tecnología convirtiéndose en un estilo de vida durante la pandemia, lo cual podría modificar bastantes actividades en la post-pandemia permitiendo nos encontremos más cerca que nunca a escenarios mixtos (presenciales combinados con virtuales). Estos deben desarrollar nuevos métodos y continuar renovando las formas tradicionales de los procesos educativos, organizacionales y sociales. Las RSD están ahí, cada una tiene distintas características y atiende a diferentes fines, es nuestra responsabilidad adaptarlas para que respondan a diversas necesidades y objetivos.

\section{Referencias}

Alárcon, D. N., Ramírez Quispe M., y Vilchez Velito M. Y. (2013). Las tecnologías de la información y comunicación (TIC) y su relación con el aprendizaje del idioma inglés en los estudiantes de la especialidad de inglés-francés, promoción 2011 de la Universidad Nacional de Educación Enrique Guzmán y Valle, Chosica, 2013 [Tesis Licenciatura, Universidad Nacional de Educación]. Repositorio UNE. https://bit.ly/2Q2wlKm

Arrivillaga, M., Salazar, I. C., y Correa, D. (2003). Creencias sobre la salud y su relación con las prácticas de riesgo o de protección en jóvenes universitarios. Redalyc, 34(4), 186-195. https://bit.ly/3viB36V

Asociación de internet. (2018). $14^{\circ}$ Estudio sobre los Hábitos de los Usuarios de Internet en México 2018. https://bit.ly/3rZxPCY

Asociación de internet. (2019). $15^{\circ}$ Estudio sobre los Hábitos de los Usuarios de Internet en México 2019. https://bit.ly/2OzBA3D

Ávila Font, E. (2003). Las nuevas tecnologías de la información y la comunicación como herramientas necesarias en la formación profesional de los estudiantes universitarios. etic@net 1, 1-5. https://bit.ly/3qQW70B

Bourdieu, P. (1991). El sentido práctico. Taurus. 
Boyd, D. M., y Ellison, N. B. (2007). Social network sites: Definition, history, and scholarship. Journal of Computer-Mediated Communication, 13(1), 210-230. https://doi.org/10.1111/j.10836101.2007.00393.x

Capdevielle, J. (2011). El concepto de habitus: "con Bourdieu y contra bourdieu". Revista Andaluza de ciencias sociales, (10), 31-45. https://bit.ly/3eQei4z, consultado el: 01/12/2020

Castaño Ramírez, A. (2011). Introducción al concepto de hábito de Charles Peirce para el comportamiento del consumidor. Punto de vista 2(3), 9-14. https://bit.ly/38EpDR9

Cobo Romaní, J. C. (2009). El concepto de tecnologías de la información. Benchmarking sobre las definiciones de las TIC en la sociedad del conocimiento. ZER- revista de estudios de comunicación. 14(27), 295-318. https://bit.ly/3bN6Eps, consultado el: 01/12/2020

Comisión económica para América Latina y el Caribe. (2020). Informe especial COVID-19 América Latina y el Caribe ante la pandemia del COVID-19. Efectos económicos y sociales. Comisión Económica para América Latina y el Caribe. https://bit.ly/3czlptY

ComScore (2021). Rankings más recientes. En: ComScore. https://bit.ly/3eJvBnP

Conti, M. y Passarella A. (2017). Online Social Networks and Media. Online Social Networks and Media. 1, 44-55. https://bit.ly/30HFMkr

Cortés Campos, R. L., Zapata González, A., Menéndez Domínguez, V. H. y Canto Herrera, P. J. (2015). El estudio de los hábitos de conexión en redes sociales virtuales, por medio de la minería de datos. Innovación Educativa, 15 (68), 99-114. https://bit.ly/2PXWrxT

Criado Grande, J. I. y Rojas-Martin F. (2015). El impacto de las redes sociales digitales en las administraciones locales. Mitos y realidades en el caso español. Revista de internet, derecho y política, (20), 25-42. https://doi.org/10.7238/idp.v0i20.2581

Gómez, R. y Sosa-Plata, G. (2012). Los Medios Digitales: México. (Country Report). Open Society fundation. https://osf.to/3bNQYT3

Guerra Manzo, E (2010). Las teorías sociológicas de Pierre Bourdieu y Norbert Elias: los conceptos de campo social y habitus. Estudios Sociológicos, XXVIII (83),383-409. https://bit.ly/30J3flq

Guerrero Montoya, L. R. y León Salazar, A. R. (2010). Estilo de vida y salud. Educere, 14(48),13-19. https://bit.ly/3liaFoR

Health Education Unit (1986). Life-styles and health. Social Science \& Medicine, 22(2), 117-124. doi: https://doi.org/10.1016/0277-9536(86)90060-2

Hernández Sampieri, R., Fernández Collado, C. y Baptista Lucio, P. (2014). Metodología de la Investigación. McGrawHill.

Hidalgo Toledo, J. A. (2014). Redes sociales digitales y sus implicaciones éticas. En Del Prado Flores, R (Eds) Ética Y Redes Sociales, (pp. 87-116). Universidad Anáhuac. https://bit.ly/2Ov1sOc

IAB México, y Televisa digital. (2018). Estudio de consumo de medios y dispositivos entre internautas mexicanos 2016 Generación Z. https://bit.ly/3tnGFuT

Incera, J., Cartas R. y Cairo O. (2007). Redes Digitales: Presente y Futuro. Laboratorio de Redes Avanzadas ITAM. https://bit.ly/3vmFQnK

INEGI. (2012). Encuesta Nacional de Consumo Cultural de México (ENCCUM). https://bit.ly/3ljyTzo

Instituto Federal de Telecomunicaciones, Instituto Federal de Telecomunicaciones, y Transportes, S. de C. y. (2019). Encuesta sobre Disponibilidad y Uso de Tecnologías de la Información y la Comunicación en los Hogares. https://bit.ly/2OoUFWq

López Jiménez I. E. y Villafañe Rodríguez C. (2011). La integración de las TIC al currículo: propuesta práctica. Razón y palabra, (74), 1-17. https://bit.ly/3czmxio

Ochoa-Ontiveros, Mélida María (2019). Trayectoria escolar y estrategias extraclase para cumplir deberes escolares. [Tesis Maestría en Innovación Educativa, Universidad Autónoma de Chihuahua. Chihuahua, México] 
Organización Mundial de la Salud (2020). COVID-19: cronología de la actuación de la OMS. https://bit.ly/3eHv3yz

Palomares Estrada, L. (2014). Estilos de vida saludables y su relación con el estado nutricional en profesionales de la salud. Tesis (Maestría en Gestión y Docencia en Alimentación y Nutrición, Universidad Peruana de Ciencias Aplicadas. Lima, Perú). https://bit.ly/30OFMPr, consultado el: $01 / 12 / 2020$

Pullen C., Walker S.N. y Fiandt K. (2001). Determinants of health-promoting lifestyle behaviors in rural older women. Family and Community Health. Family \& Community Health. 24(2), 49-72. doi: 10.1097/00003727-200107000-00008

Sanabria-Ferrand, P. A., González Q., L. A. y Urrego M., D. Z. (2007). Estilos de vida saludable en profesionales de la salud colombianos: estudio exploratorio. Revista Med, 15(2), 207-217. https://bit.ly/3czlMpy

Sánchez Duarte, E. (2008). Las tecnologías de información y comunicación (TIC) desde una perspectiva social. Revista electrónica educare, 8, 155-162. https://bit.ly/30HExBN, consultado el: $01 / 12 / 2020$

Similarweb (2020). Ranking de aplicaciones más utilizadas. SimilarWeb https://bit.ly/3lnit91

Tavera Uribe, I. C. (2018). Motivadores e inhibidores de los millennials con independencia económica para la compra de alimentos saludables como frutos secos y semillas a través de medios en línea [Tesis Maestría, Universidad EAFIT]. Repositorio UNE. https://bit.ly/3rOiigg

Torres Nabel, L. C. (2013). Los mecanismos de lo político en las redes sociales de Internet. Aposta, 58, 127. https://bit.ly/3qNDYAQ

Winocur Iparraguirre, R. y Sánchez Martínez, J. A. (2015). Redes sociodigitales en México. Fondo de Cultura Económica.

Zapata O. (2005). La aventura del pensamiento crítico. Herramientas para elaborar tesis e investigaciones socioeducativas. Pax México

\section{Semblanza de los autores}

Rubén Cervantes Hernández. Candidato a doctor en Educación, Artes y Humanidades por la Universidad Autónoma de Chihuahua. Maestro en Investigaciones Humanísticas y Educativas en Desarrollo Humano y Cultural, con Mención Honorífica en la defensa de grado con la tesis: "La política cultural y su impacto en la juventud zacatecana actual" por la Universidad Autónoma de Zacatecas. Licenciado en Negocios Internacionales por la Universidad Politécnica de Zacatecas.

Paola Margarita Chaparro-Medina. Doctora en Filosofía con Acentuación en Estudios de la Cultura por la Universidad Autónoma de Nuevo León (UANL), Máster en Sociología por la Universidad de Artes y Ciencias Sociales de Santiago de Chile y Licenciada en Ciencias Políticas por la UANL. Es catedrática de Tiempo Completo en el Doctorado en Educación, Artes y Humanidades de la Facultad de Filosofía y Letras de la Universidad Autónoma de Chihuahua. 